\title{
Médecins en uniforme et uniformité de la médecine : carrière et identité du médecin des armées
}

\author{
Marc Loriol, Université de Paris I
}

Sciences Sociales et santé, mars 1999, n ${ }^{\circ}$, XVII, pp. 5 à 34.

\begin{abstract}
Résumé: Les médecins des armées occupent, en France, une position atypique aussi bien dans le monde militaire qu'au sein de la profession médicale. En réaction à cette position, certains d'entre eux peuvent être amenés à adopter des représentations particulières de ce que doit être une bonne pratique médicale, inversant les valeurs habituellement prônées par la médecine dominante (activité libérale, primat du biologique...). Cela rapproche une partie significative des médecins des armées d'une forme plus générale de pratique que l'on peut qualifier de "médecine sociale". Quelles conditions vont favoriser ce type de stratégie identitaire, atypique au sein de la profession médicale? Deux moments sont particulièrement importants dans ce processus : la première expérience en médecine d'unité et le retour à la vie civile. C'est à cette phase de la carrière du médecin des armées que se dévoilent avec le plus de netteté les liens entre les caractéristiques propres à la médecine militaire et l'orientation pratique et cognitive en faveur d'un certain type d'exercice de la médecine, stigmatisé par la pratique dominante. Le choix, effectué sous contraintes, de tel ou tel secteur de reconversion détermine alors le type de stratégie identitaire préférentiellement mené : rattachement aux valeurs dominantes dans la médecine civile ou remise en cause de ces valeurs et construction d'une identité spécifique de médecin militaire.
\end{abstract}

Summary : In France, military doctors have an atypical position within the military world as well as in the medical profession. As a reaction to this atypical position, some of them have a certain attitude about what is considered "correct" medical practice and they inverse the common values of most members of the modern medical profession (independent practice, physiological understanding of disease...). Certain military doctor who take this inversed position could be qualified as doctors of social medecine. What conditions encourage this type of process atypical of the majority in the medical profession? Two points in time are especially important in this process: first experience as a doctor in a military base, together with the moment when the individual returns to civil life. This is the moment in a military doctor's career when the relationship between typical military medical tendencies and practical, cognitive orientation towards a certain type of medical practice wich is stigmatized by most medical practionners becomes clearly immasked. The choice, wich is limited to certain sectors of reorientation, determines the identity defining strategy the military doctors uses: either he remains attached to the dominant values identifying civil medecine or he questions these values and builds is own identity within specific values of military medecine.

Résumé en espagnol: Los médicos militares ocupan, en Francia, una posicion atipica tan bien en el mundo militar que en la profesion médical. En reaccion de esta posicion, algunos de entre ellos pueden ser amenazados a adoptar representaciones particulares de lo que deve ser una buena practica médical, inversando los valores habitualmente adelantados por la médecina dominante (actividad libérad, importancia del biológico...). Esto acera una parte significativa de los médicos militares de la médicina social. Quales condiciones van an a favorizar este tipo de estrategia identitaria, atipica en le profesion médical ? Dos momentos son particulamente importantes en este procedimiento : la primera experiencia en medicina de cuartel militar y la vuelta a la vida civil. Es en este momento de la carera que se puede observar los vinculos entré las particularides de la médicina militar y una practica médical marginal. El sector de reconversion esligido determina la estrategia identitaria : adopcion de los valores dominantes de la medicina o identitad específica de médicos de las armadas.

Mots clés: Médecin militaire, identité professionnelle, carrière, reconversion. 
La sociologie fonctionnaliste des professions, principalement quand il s'agit de la médecine, tend à considérer le groupe professionnel comme un acteur homogène partageant un ensemble minimal de pratiques, de valeurs et d'intérêts communs ; cette homogénéité étant liée au contrôle par la profession du système de formation. C'est en réaction à cette vision unifiée des professions que les auteurs interactionnistes ont mis l'accent sur l'importance des différenciations internes. Ainsi, pour rendre compte de la diversité de la profession médicale, Anselm Strauss (1992) utilise la notion de segment qui désigne l'émergence des divers groupements de fait ne se confondant pas avec les différentes spécialités médicales. Pour cet auteur, un segment peut être distingué grâce à plusieurs critères : le type de relation avec la clientèle, la méthodologie et les techniques utilisées, les intérêts particuliers, les relations de confraternité et l'image publique que chaque segment cherche à donner de la profession. Chaque segment développe donc une identité professionnelle-c'est-à-dire une manière particulière d'interpréter le rôle et l'activité du professionnel - qui lui est propre.

L'opposition entre une vision unifiée de la profession et la description des différents segments qui existent en son sein occulte cependant la question des écarts aux normes dominantes : comment les professionnels qui exercent selon des modalités atypiques vivent ces différences? Quelles représentations de leur pratique vont-ils adopter et diffuser? Ces questions sont particulièrement importantes dans le cas de la médecine où il existe un modèle dominant particulièrement fort, imposé par certains segments de la profession et structuré autour de deux principes centraux : supériorité de la pratique libérale et du paiement à l'acte d'une part et primat de l'approche biologique et physiologique de la maladie, par rapport aux approches psychologiques ou sociales, d'autre part ${ }^{1}$. Comment les médecins que leur situation particulière conduit à s'écarter, dans leur pratique, de l'un ou l'autre de ces principes vont se positionner par rapport à ce modèle dominant? Michel Arliaud (1981), reprenant la notion de champ de Pierre Bourdieu, montre comment la dissidence par rapport au modèle médical dominant est favorisée par une position marginale dans la profession médicale : les dominés, dans un champ, ont intérêt à une remise en cause symbolique des principes qui déterminent la hiérarchie interne à ce champ. Ainsi, les généralistes, de plus en plus perçus comme des nonspécialistes et dont le travail porte de moins en moins sur des problèmes d'ordre strictement biologique et physiologique ont toutes les chances d'être parmi les premiers à remettre en cause la pensée médicale dominante. Plusieurs travaux ont d'ailleurs fait état de cette corrélation entre marginalité et choix d'une pratique médicale alternative (Bouchayer, 1984 ; Chateauraynaud, 1986 ; Traverso, 1993 ; etc.).

\footnotetext{
${ }^{1}$ La façon dont ce modèle s'est imposé historiquement ne constitue pas l'objet de ce travail. Pour le cas de la France, voir, par exemple, Foucault (1963), Ferrand-Nagel (1993) ou Hassenteufel (1997).
} 
Mais, comme le note Michel Arliaud lui-même, tous les omnipraticiens ne deviennent pas des dissidents et certains font même preuve d'un grand conformisme. De même, une recherche récente (Piotet et al., 1997) sur les médecins du travail, sous-ensemble encore plus marginalisé que celui des omnipraticiens, montre que si certains d'entre eux cherchent à donner un sens positif à leur pratique spécifique à partir d'une défense de l'idée de prévention par l'amélioration des conditions de travail, d'autres semblent au contraire entériner leur statut dominé. Par ailleurs, seuls 53\% des 453 médecins du travail ayant répondu à cette enquête "ont un fort sentiment d'appartenance à une profession spécifique". Pourquoi certains généralistes ou médecins du travail, mais pas tous, vont chercher à construire pour eux-mêmes et pour autrui une image particulière de leur rôle et de leur activité remettant partiellement en cause les principes dominants dans la profession médicale? Quels sont les facteurs qui favorisent la constitution d'une identité professionnelle spécifique et alternative à la forme dominante? Pour Michel Arliaud (1981), la réponse serait à rechercher dans les expériences sociales antérieures à la socialisation professionnelle, dans le "déracinement social (consécutif à une mobilité sociale ou géographique des familles)". Si cette hypothèse est intéressante, elle est probablement insuffisante dans le sens où elle exclut de l'analyse toutes les expériences déterminantes vécues lors de la vie professionnelle elle-même. Une étude rétrospective auprès de 5491 médecins retraités inscrits au fichier de Conseil de l'Ordre (Herzlich et al., 1993) a montré l'existence d'une grande cohérence entre les choix de carrière et les contraintes professionnelles et sociales. Parmi les médecins généralistes de l'échantillon, une distinction a pu être faite entre les "généralistes de ville" (33\% de l'échantillon) et "l'élite des généralistes" $(6 \%)$. Le premier groupe, qui s'est révélé sociologiquement très proche de celui des médecins salariés $(17 \%)$, est caractérisé par un même type de parcours : origine sociale, choix de carrière du fait des contraintes financières ou de réussite universitaire, passage d'un groupe à l'autre, etc. Le second groupe, qui a exercé moins longtemps une pratique générale, s'est révélé plus proche des spécialistes de ville (par l'implantation sociale et géographique, la participation aux différents centres de pouvoir, politique, syndical ou professionnel...). Les oppositions simples entre spécialistes et généralistes ou entre médecins libéraux, salariés et hospitaliers ne sont pas suffisantes pour comprendre les prises de position sur le conventionnement, le mouvement de spécialisation biomédical ou l'importance du psychologique dans la relation au malade. Seule la complexité des parcours permet de comprendre comment se forgent les identités.

Pour Hugues (1958), l'identité professionnelle s'élabore à travers quatre éléments : la nature de la tâche, la conception du rôle, l'anticipation des carrières et l'image de soi. A cela, il faut ajouter les relations de pouvoirs, aussi bien au niveau de l'entreprise (Sainsaulieu, 1977) que de la profession. Comment ces différents éléments vont peser pour entraîner certains 
médecins exerçant dans des cadres professionnels atypiques à adopter une identité spécifique et positive?

L'exemple des médecins militaires semble particulièrement pertinent, dans le cadre d'un tel questionnement, pour au moins trois raisons : Il s'agit tout d'abord d'un sous-ensemble professionnel qui n'a pas encore ${ }^{2}$ attiré l'attention des sociologues malgré son importance relative : en terme d'effectifs à temps plein, ils ont été, durant ces cinquante dernières années, juste un peu moins nombreux que les médecins du travail ou les médecins consei1 ${ }^{3}$. En 1995, on compte 2647 médecins des armées d'active sur un effectif de 16750 médecins salariés (hors établissements hospitaliers), parmi lesquels 6300 médecins du travail ou encore 1255 médecins de $\mathrm{PMI}^{4}$. Les médecins des Armées représentent donc un sous-groupe important parmi les médecins salariés. Ensuite les médecins militaires présentent un grand nombre de particularités qui les placent dans une situation atypique par rapport aux normes dominantes de la pratique médicale : formation spécifique lors de la dernière année, médecine salariée, pluralité des tâches, participation au service public, non-inscription au conseil de l'ordre, etc. Cette situation s'accompagne une image de marque plutôt médiocre ${ }^{5}$ de la médecine militaire - à l'exception des grands hôpitaux comme le Val-de-Grâce - en partie entretenue par certains médecins civils pour qui l'existence même d'un groupe de médecins salariés ne consacrant qu'une partie de leur temps aux soins constitue une menace potentielle pour le statut de praticien libéral. Les médecins militaires ont relativement conscience de cette situation et sont amenés à se positionner par rapport aux normes dominantes et à réaffirmer leur identité de médecin. Enfin, la plupart des médecins des armées terminent leur carrière par une reconversion dans la médecine civile qui viendra révéler, par la confrontation nécessaire avec les normes dominantes qu'elle entraîne, l'identité professionnelle construite lors de l'expérience militaire.

L'objet de cet article est donc de comprendre quels sont les éléments qui vont favoriser chez le médecin des armées, tout au long de sa carrière militaire et éventuellement civile, une attitude positive à l'égard de conditions d'exercice jugées plutôt négativement par la plupart des médecins civils, à l'exception de quelques représentant d'autres groupes de médecins

\footnotetext{
${ }^{2}$ Du moins à ma connaissance.

${ }^{3}$ Comme le suggèrent les données présentées par Herzlich et al. (1993), qui de plus sous-estiment le nombre des anciens médecins militaires puisque seuls les médecins inscrits au conseil de l'ordre ont été enquêtés.

${ }^{4}$ Sources : Sirpa Santé ; Annuaire des statistiques sanitaires et sociales, 1997, Ministère du Travail et des Afaires sociales et Le Monde du 02/10/1996.

${ }^{5}$ Il n'est pas question d'analyser ici l'image, dans l'opinion, des médecins des Armées. Il est possible de rappeler simplement qu'une expression courante pour signifier la différence entre deux choses, $\mathrm{A}$ et $\mathrm{B}$, consiste à dire : "A est à $\mathrm{B}$ ce que la médecine militaire est à la médecine". Si il n'est pas facile de distinguer, dans cette formule, également utilisée pour la musique, ce qui relève de la stigmatisation du statut de militaire de ce qui relève de celle de la médecine salariée, l'image médiocre dans l'opinion, notamment celle des praticiens libéraux, des médecins du travail ou des médecins scolaires peut laisser penser que la deuxième hypothèse n'est pas dénuée de tout fondement.
} 
salariés et de certains généralistes. Deux moments apparaissent particulièrement déterminants dans la carrière du médecin militaire pour la construction de son identité professionnelle : le passage par la médecine d'unité (c'est-à-dire dans une caserne, une base aérienne ou à bord d'un navire) et la reconversion dans la médecine civile. Quels mécanismes cognitifs et sociaux sont à l'oeuvre à chacune de ces deux étapes et quelles conditions sociales permettent de rendre compte de l'adoption, ou non, d'une identité professionnelle spécifique fondée sur le renversement des stigmates - au regard des critères imposés par la médecine libérale (supériorité du paiement à l'acte) du ou hospitalière (plus grande scientificité de la médecin organique) - de la médecine militaire ?

Pour tenter d'apporter quelques éléments de réponse à cette question, deux recherches quantitatives seront particulièrement utilisées : une enquête auprès de 562 médecins d'unité sur leurs conditions de travail, réalisée en octobre 1992 par le Conseil de la Fonction Militaire du Service de Santé des Armées et surtout une enquête réalisée en janvier 1994 à l'Observatoire Social de la Défense (Loriol, Rio, 1994) sur "le retour à la vie civile des militaires ayant acquis droit à pension", parmi lesquels 112 médecins ayant quitté les armées en 1990, 1991 ou 1992; enquête à laquelle l'auteur a participé dans le cadre d'un service militaire en tant que scientifique du contingent en 1993-19946. Ces deux enquêtes comportent des questions fermées et une (deux pour la deuxième) question ouverte. Dans ce texte, il sera fait allusion à la première comme "l'enquête 1992" et la deuxième comme "l'enquête 1994". Les extraits de libre réponse seront donnés en italique et notés "LR.92" pour celle de "1'enquête 1992" et "LR1.94" ainsi que "LR2.94" pour, respectivement, la première et la deuxième libre réponse de "l'enquête 1994"7. Pour affiner l'interprétation des données quantitatives et apporter quelques éléments supplémentaires sur les spécificité de la pratique médicale en milieu militaire, seront également utilisées des discussions personnelles avec plusieurs médecins militaires ou ancien médecins militaires ${ }^{8}$ et une infirmière militaire, portant notamment sur les premières hypothèses qui ont conduit à ce travail. Parmi ces discussions, celles répétées avec le médecin-chef qui fut mon supérieur direct lors de mon service national, ont été particulièrement instructives. Enfin, la lecture rétrospective de la revue Médecine et Armées à partir de 1981, date à laquelle s'arrête la collection que j'ai pu consulter, et jusque fin 1996, a apporté quelques éléments sur la nature des recherches menées dans le cadre de la médecine militaire. Cet éclectisme des sources est lié d'une part à l'absence

\footnotetext{
${ }^{6}$ Lors de la rédaction du questionnaire destiné aux anciens médecins militaires, la problématique de l'identité professionnelle atypique était déjà esquissée, d'où la présence de questions s'y rapportant. De plus, de nombreux tris croisés et analyses secondaires, y compris des analyses factorielles, ont pu être menés spécifiquement pour ce travail. Si le faible effectif (112 médecins) impose d'être prudent quant aux résultats quantitatifs des tris croisés, un taux de réponse de $50 \%$ permet de postuler une certaine représentativité.

${ }^{7}$ LR.92 et LR2.94 proposaient aux médecins interrogés d'apporter d'éventuels commentaires complémentaires sur des points qui n'auraient pas été, ou pas suffisamment, traités dans l'enquête. LR1.94 portait sur les différences perçues entre la pratique militaire et la pratique civile.

${ }^{8} 6$ médecins en tout ont été rencontrés, dont certains à plusieurs reprises, trois entretiens ont été enregistrés.
} 
de données et d'analyses précises sur les médecins des armées et d'autre part à la volonté de cerner par plusieurs approches cet objet complexe que représente la notion d'identité professionnelle?.

\section{I. - La carrière militaire : l'expérience déterminante mais paradoxale de la médecine d'unité}

Lors de sa carrière dans l'Armée, principalement au cours de sa première expérience en unité, le médecin militaire est confronté à des routines, des conditions de travail et des tâches qui peuvent s'écarter plus ou moins nettement de ce que rencontre habituellement le médecin civil. Ces différences sont parfois la source d'une image dévalorisée de la pratique militaire jugée, par le sens commun, moins fiable, moins scientifique. Face à ces contraintes, certains médecins militaires, notamment ceux qui ne peuvent s'appuyer sur la légitimité que procure la possession d'une spécialité valorisée dans le civil, développent des prises de position favorables à la pratique salariée et à la prise en compte par la médecine de problèmes non strictement organiques. Cette prise de position s'inscrit dans l'organisation et l'orientation globale du Service de Santé des Armées, mais elle constitue également une réponse individuelle d'un grand nombre de médecins des armées à leur déviance de fait par rapport aux normes dominantes dans la profession médicale.

\section{1 - Le travail du médecin au sein du Service de Santé des Armées (SSA)}

Dès 1708, en France, est créé un service de santé stable et permanent avec des infirmeries dans chaque place forte. Mais ce n'est qu'avec les lois de 1882 et 1889 que le SSA s'autonomise de la tutelle de l'administration militaire et que la responsabilité de son fonctionnement revient, à tous les échelons hiérarchiques, à un médecin. En 1986, le SSA compte 2800 médecins, officiers d'active, et 1350 médecins appelés du contingent. En 1995 ces effectifs sont passés respectivement à 2650 et 1450 . La future suppression du service national n'aura, semble-t-il, pas d'influence sur le nombre des médecins militaires d'active, la défection des médecins du contingent étant compensée par la réduction de la charge de travail. Au niveau des infrastructures le SSA comptait en 199032 hôpitaux dont 9 hôpitaux des armées (notamment le Val-de-Grâce), 18 centres hospitaliers (l'équivalent des hôpitaux généraux civils), 4 hôpitaux thermaux et l'infirmerie-hôpital de Dakar ${ }^{10}$. Dans chaque formation de l'armée de terre, de l'armée de l'air, de la gendarmerie et de la marine, un médecin chef est responsable de l'exécution du service médical de l'unité.

\footnotetext{
${ }^{9}$ Je tiens à remercier ici les professeurs Claudine Herzlich et François Gresle pour leurs remarques sur des versions antérieures de ce travail.

${ }^{10}$ Source : Sirpa Santé.
} 
Les missions du médecin militaire sont nombreuses : expertise, sélection, recherche, prévention, enseignement et évidemment médecine de soins. Les médecins du SSA sont payés en fonction de leur grade et de leur affectation et non en fonction de la quantité d'actes effectués. Les visites médicales en unité sont gratuites pour tous les militaires (appelés et engagés, soit 931000 personnes en 1993, OSD, 1993). Les 301000 appelés du contingent sont des "ayants-droit obligatoires", c'est-à-dire qu'ils ne sont plus couverts par l'Assurancemaladie et qu'ils doivent nécessairement s'adresser au SSA. Les militaires de carrière et sous contrat doivent s'adresser à un médecin militaire pour les maladies et accidents liés au service (Brumter, 1979). Pour les autres problèmes de santé, ils sont libres de consulter, pour eux ou leur famille, un médecin civil ou un médecin militaire. Plusieurs facteurs peuvent jouer en faveur du choix du second : l'isolement géographique, les relations personnelles et la gratuité de la médecine d'unité. Entre le médecin et son patient peut alors se créer, dans certains cas, un univers symbolique spécifique dans lequel l'absence de paiement à l'acte est perçu par les deux parties comme le signe d'un désintéressement matériel et d'une meilleure qualité du soin ${ }^{11}$. Le dogme de la supériorité du paiement à l'acte, imposé par le libéralisme médical, est donc inversé.

En outre, un certain nombre de médecins d'unité doivent, sous l'appellation de "médecin de prévention", assurer le rôle de médecin du travail auprès des 113000 civils de la défense et des arsenaux (Dyevre et Giacomoni, 1995). La médecine militaire est donc totalement fonctionnarisée, sans relation marchande avec sa clientèle et pour une grande part consacrée à des tâches de prévention, de gestion de la main-d'oeuvre et de médecine du travail. Cet aspect multiforme du travail du médecin d'unité permet l'administration sanitaire au sein d'une "institution totale" (selon le terme de Goffman, 1968) d'une population nombreuse, ce qui rend possible la mise en place d'une véritable médecine collective (Gattef, 1986), y compris dans ses risques de "dérive".

L'exemple extrême de ce qui pourrait être considéré comme une "dérive" serait le triste épisode des expériences menées par l'armée américaine sur les effets du LSD. En France la question se pose avec certaines pratiques comme les tests de dépistage du sida "imposés" dans certains cas ; ou les consultations obligatoires avec un psychiatre pour tous les militaire ayant participé aux opérations de 1994 au Rwanda. De façon plus globale, c'est la question du "volontariat", pour des études médicales de toutes sortes menées auprès de militaires, engagés ou appelés, qui pourrait être problématique dans les armées ${ }^{12}$. La question de l'éventualité d'une "dérive" de la médecine militaire, qui consisterait à faire passer les besoins de

\footnotetext{
11 Un regret parfois exprimé par les anciens militaires, non médecins, est la perte de l'accès à la médecine militaire perçue comme plus humaine car moins mercantile (Loriol, Rio, 1994), certains cherchent même à consulter d'anciens médecins militaires installés en libéral (entretien avec un ancien médecin militaire, ORL). ${ }^{12}$ Source : discussion personnelle avec une infirmière militaire.
} 
l'institution employeuse ou de la recherche avant ceux du malade, est particulièrement sensible. Les tenants de la médecine libérale ont en effet très souvent utilisé ce type d'argument pour préserver leur statut de travailleurs indépendants. Présenter les médecins militaires ou pénitentiaires comme étant avant tout soucieux des besoins de leurs institutions respectives ou accuser le médecin du travail de n'être qu'un instrument de la politique patronale est surtout un moyen de défendre la médecine libérale. Une partie de l'image négative de la médecine militaire relève probablement de ce type d'explication. Les médecins militaires rencontrés, tout comme de nombreux médecins du travail, sont conscients de ce type de critique qui les incite à réaffirmer fortement leur identité de médecin et, pour les plus engagés d'entre eux, à proposer leur propre construction de ce qui serait "le besoin du malade", fondée sur la notion de "médecine globale".

Ce type d'approche se retrouve dans les productions intellectuelles d'un certain nombre de médecins militaires. Le dépouillement systématique, de 1981 à 1996, de la revue Médecine et Armées montre que cette revue accepte régulièrement dans ses colonnes des travaux sur des questions qui débordent le simple cadre de la médecine organique. Les plus nombreux sont ceux qui concernent les problèmes liés à l'alcoolisme (plus de 30 articles dont un numéro spécial) et dans une moindre mesure le tabagisme et la toxicomanie ( 8 articles). Viennent ensuite les études sur la résistance aux situations extrêmes, le stress et la fatigue (22 articles) ; les questions de gestion des ressources humaines (motivation, absentéisme, désertion, relations de pouvoir etc., soit 15 articles) puis les problèmes de suicides et de délinquance ( 8 articles). D'autres thèmes récurrents peuvent être cités: économie et droit de la santé, ergonomie, ethnomédecine (ici, l'étude des problèmes particuliers posés par les croyances et les pratiques médicales indigènes pour la pratique de la médecine occidentale dans certains pays du tiers monde), etc. La présence de ce type d'article parmi des recherches consacrées à des questions strictement biologiques et physiologiques revient à élever, au moins sur un plan symbolique, les études sur des problèmes comportementaux et psychosociaux au rang de sujets légitimes pour la recherche médicale. Il est possible supposer, même si cela ne peut pas être démontré ici de façon rigoureuse, que la lecture régulière de Médecine et Armées, ou au moins de son sommaire ${ }^{13}$, renforce chez certains médecins militaires une représentation d'un champ d'intervention et d'investigation médicale plus large que l'approche strictement physiologique imposée par la médecine hospitalière. Cette revue à caractère scientifique ${ }^{14}$ permet également à quelques médecins d'unité d'utiliser leur situation particulière pour faire de la recherche et donc valoriser leur position marginale.

\footnotetext{
${ }^{13}$ Cette revue, publiée à huit numéros par ans, est distribuée auprès de tous les médecins militaires.

${ }^{14}$ Les informations sur la vie du SSA et ses aspects administratifs sont publiées à part, dans un autre périodique, afin de ne pas brouiller l'aspect scientifique de la revue.
} 
2- La première expérience en médecine d'unité : une étape significative dans la construction de l'identité

L'entrée dans la carrière de médecin militaire se fait par l'intégration de l'une des deux écoles du SSA (de Lyon et Bordeaux) sur concours après le baccalauréat. Quels critères peuvent déterminer le choix de la médecine militaire plutôt que celui de la médecine civile ? L'argument d'un accès plus facile doit être tout d'abord rejeté au regard des taux d'admissions (en 1986, 103 places pour 2600 candidats) qui sont comparables aux numerus clausus imposés à la fin de la première année des études civiles. La rémunération lors des études peut orienter le choix des candidats les plus modestes et représente un élément de démocratisation des études médicales que leur longue durée rend particulièrement coûteuses. Mais l'élément le plus significatif est probablement l'intérêt et la sympathie pour les conditions de travail propres au milieu militaire. D'ailleurs, comme les autres officiers, les médecins des armées sont beaucoup plus souvent fils de militaires que leurs collègues civils. La possibilité d'accès aux études longues pour des candidats d'origine modeste et un a priori favorable à l'égard de l'organisation bureaucratique de l'Armée pourraient alors expliquer pourquoi l'identité professionnelle compatible avec l'exercice salarié et l'ouverture aux questions psychosociales serait plus répandue parmi les médecins militaires que parmi les médecins civils. La socialisation primaire serait alors congruente avec la socialisation professionnelle qui se réalise lors des premières expériences professionnelles.

Mais quelques éléments partiels semblent indiquer que les représentations et l'identité des jeunes élèves des écoles du SSA ne sont pas très éloignées de celles des étudiants en médecine. Ainsi, une enquête d'opinion (Cristau, 1989) menée auprès des élèves de l'école de santé des armées de Lyon sur l'alcoologie, domaine plutôt marginal de la médecine, a montré une grande similitude d'opinion quant à la réticence vis-à-vis de la matière, avec des étudiants civils. Ce n'est qu'après l'année en école d'application et surtout après l'expérience de la médecine d'unité que le médecin des armées acquiert une opinion plus positive à l'égard de cette discipline. C'est donc bien la première expérience professionnelle qui joue un rôle déterminant dans la formation d'une conception de la médecine favorable à la médicalisation d'un problème social comme l'alcoolisme. Isabelle Baszanger (1983), dans une étude sur les médecins généralistes, avait souligné également l'importance de la première expérience professionnelle dans la socialisation au métier et la constitution des représentations. Les approches intéractionnistes et fonctionnalistes sont alors, en quelque sorte, dépassées au profit d'une voie médiane: c'est la nécessité de s'adapter aux contraintes et aux spécificités de la 
première expérience professionnelle, souvent très différente de l'idéal présenté lors des études, qui est à l'origine de représentations et d'attitudes spécifiques ${ }^{15}$.

Après sept années d'études, dont le programme est équivalent à celui des facultés de médecine civiles, les élèves médecins doivent effectuer une année complémentaire à l'école d'application du Val-de-Grâce où sont particulièrement enseignées les connaissances plus spécifiques à la médecine militaire. Cette dernière année et le fait d'être rémunéré et isolé des médecins civils contribuent sans doute à favoriser l'éclosion d'un sentiment d'identité spécifique de médecin des armées ${ }^{16}$. Ensuite, tous, y compris ceux qui souhaitent se spécialiser, doivent passer au moins trois ans à exercer dans une unité (c'est-à-dire une caserne, une base aérienne ou embarqué sur un navire). Le passage par la médecine d'unité constitue la première expérience professionnelle et la plus marquante, avec les expériences outre-mer, comme il est possible de le comprendre à la lecture des libres-réponses de "l'enquête 1994". Le travail du médecin d'unité est, pour une grande part, différent de celui du médecin libéral ou hospitalier civil. D'après "l'enquête 1992", les médecins d'unité disent consacrer $22 \%$ de leur temps à la médecine de soin, $30 \%$ à la médecine d'expertise et de prévention, $12 \%$ au soutien médical, $20 \%$ aux activités administratives, $9 \%$ aux activités militaires et $7 \%$ aux autres activités. Il serait probablement intéressant de comparer ces chiffres avec des données équivalentes pour les médecins civils mais il n'existe pas de découpages semblables dans les études sur les autres catégories de médecins ${ }^{17}$. Ces dernières, en effet, distinguent plutôt le temps passé en visite et consultation des autres activités. Il n'est pas possible de savoir si le temps de visite est consacré au soin ou à l'expertise médicale. Le fait de passer plus de temps à l'expertise et à la prévention qu'aux soins proprement dits distingue néanmoins le médecin militaire de son confrère libéral.

Le rôle du médecin d'unité est donc multiple et comprend également certains aspects plus informels : gestion de conflits personnels ou hiérarchiques, aide dans certains problèmes psychologiques et/ou sociaux. Un exemple typique est peut-être celui où le médecin d'unité sert de médiateur dans les conflits qui opposent un sous-officier et sa hiérarchie qui lui reproche son alcoolisme. Au delà de son simple rôle d'expertise, le médecin est parfois sommé de trouver un accord, à propos des mutations et de l'avancement au nom de la

\footnotetext{
15 Pour Françoise Bouchayer (1994), les jeunes médecins généralistes, dans l'élaboration de leur identité professionnelle, procèdent par essais et erreurs jusqu'à ce qu'ils trouvent une certaine adaptation à leur situation sur le marché de l'offre de soin.

${ }^{16}$ Depuis 1997, le SSA a du faire appel à des médecins civils thèsés et à des officiers de réserve pour compenser une baisse du nombre de postes proposés à l'entrée à l'école. Ainsi, En 1998, il est prévu de recruter 25 médecins thèsés et 15 officiers de réserve, sur un total d'environ 120 recrutements. Ce changement de politique peut contribuer, à terme, à une transformation de l'identité du médecin des armées.

${ }^{17}$ Par exemple, les médecins du travail disent passer $62,5 \%$ de leur temps en visites, $5,9 \%$ en travail administratif, $17,3 \%$ en activité en milieu de travail, $4,9 \%$ en formation, $5,5 \%$ en réunion, $4 \%$ en documentation personnelle et autre (Piotet et al., 1997).
} 
neutralité scientifique de son savoir, alors qu'en fait le résultat n'est qu'une simple transaction : le sous-officier est muté dans un poste plus avantageux ailleurs (ainsi la hiérarchie locale se débarrasse de lui) en échange d'une promesse de sa part de se tenir tranquille pendant quelques temps afin de ne pas donner l'impression que l'on a surtout cherché à se débarrasser de lui. Si certains médecins acceptent ce type de tâche, d'autres cherchent à l'éviter ${ }^{18}$. La présence dans la revue Médecine et Armées d'articles sur l'obéissance, la désertion ou l'absentéisme est un autre signe de la volonté de certains médecins militaires de s'investir dans des activités de régulation des problèmes sociaux. A la fois médecin du travail, médecin traitant, conseiller en gestion des ressources humaines, le médecin militaire peut ainsi avoir une action sur les personnes, leur orientation professionnelle, leur environnement physique et social. Il est donc relativement bien placé pour pratiquer une médecine plus "globale".

Ce n'est qu'après un minimum de trois ans en unité que ceux qui le souhaitent peuvent passer le concours d'assistant pour suivre des stages de spécialisation dans un hôpital d'instruction. La pratique spécialisée en hôpital militaire est équivalente à celle en l'hôpital civil. Certains médecins militaires effectuent d'ailleurs leur stage de spécialisation dans des hôpitaux civils. Les grands hôpitaux militaires ne souffrent pas de la même image négative que la médecine d'unité ${ }^{19}$. La progression dans la hiérarchie médicale au sein de la direction du SSA dépend de ces concours de spécialisation. Dans les faits, donc, les critères d'excellence imposés par la médecine hospitalière restent la condition d'une carrière réussie. Pourtant, dans leurs prises de positions officielles, les différents responsables du SSA ont, depuis 1888 (Forissier, 1988), presque toujours souligné l'intérêt d'une certaine ouverture vers les thèmes chers à la médecine sociale. Ainsi, en 1982, le directeur central du SSA se prononce pour la promotion d'une médecine tournée vers la prévention, la prise en charge de la personne globale, l'intégration "des connaissances psychosociales, des composantes épidémiologiques et socio-économiques" (Tournier-Laserre, 1982, p. 376). Si l'obtention d'une spécialité médicale comparable aux spécialités civiles permet au spécialiste militaire de se démarquer de la situation dévalorisée du médecin d'unité, l'attachement à un discours général sur la nécessité d'une médecine globale lui apporte un vernis humaniste lui permettant de dépasser ses derniers complexes d'infériorité face aux spécialistes civils, implicitement accusés de mercantilisme et de réductionnisme biologique. Ainsi, dans "l'enquête 1994", les médecins interrogés étaient amenés à choisir entre deux définitions de la médecine :"Etude des phénomènes biologiques au sens strict" ou "prise en compte de l'individu dans toutes ses dimensions, y compris psychologiques, sociales et politiques". 96,5\% ont choisi la deuxième définition et seulement $1,75 \%$ la première $(1,75 \%$ ne s'est pas prononcé). La spécialisation ultérieure n'a donc pas modifié la représentation proclamée de la mission du médecin. Si cette

\footnotetext{
${ }^{18}$ Source : discussion personnelle avec un médecin militaire, généraliste.

${ }^{19}$ Lors de mon service national j'ai d'ailleurs observé à plusieurs reprises des civils cherchant à utiliser leurs relations pour faire admettre un membre de leur famille à l'hôpital du Val-de-Grâce.
} 
situation permet au spécialiste militaire de développer une bonne image de soi et une identité positive, elle place le médecin d'unité dans une situation paradoxale: valorisé dans les discours, il reste finalement au bas de la hiérarchie du SSA. Dans ces conditions, la construction d'une image de soi positive reste problématique.

\section{3- L'identité du médecin d'unité : entre renversement du stigmate et opposition à la} hiérarchie du SSA

D'après "l'enquête 1992", un peu moins de la moitié des médecins interrogés jugent leur pratique médicale passable $(25,8 \%)$ ou décevante $(17,1 \%)$, mettant en cause notamment la lourdeur des tâches administratives et le mode de rémunération. De plus, certaines valeurs dominantes de la profession médicale restent très présentes. A la question "à quelle activité souhaiteriez-vous consacrer le plus ou le moins de temps", 50,5\% des médecins d'unité répondent désirer consacrer moins de temps aux tâches administratives et $74,1 \%$ plus de temps aux soins. Les libres réponses renforcent cette impression (rejet du travail administratif, souhait de pouvoir, dans certains cas, recevoir des honoraires...). Le travail administratif, et dans une moindre mesure la rémunération salariée, apparaissent, face à la puissance des valeurs de la médecine libérale, comme des stigmates difficiles à gérer, même pour ceux qui arrivent à construire une identité positive. Si les médecins d'unité développent, surtout ceux qui envisagent une longue carrière militaire, une assez bonne image de leur pratique médicale spécifique (57,1\% jugent leur pratique excellente ou bonne) et une identité professionnelle positive, ils restent conscients que cette image pour soi n'est pas forcément celle qui est perçue à l'extérieur. Seuls 10,9\% estiment que "l'image de la médecine d'unité est en général plutôt bonne" contre 41,1\% "plutôt mauvaise". Ce décalage explique la difficulté qu'il peut y avoir à gérer certains stigmates et à présenter comme positifs les écarts à la pratique dominante de la médecine. Imposer une pratique alternative et marginale comme supérieure nécessiterait en fait la mise en place d'un véritable mouvement social en faveur de la spécificité de la médecine d'unité qui, pour l'instant, n'existe pas. D'autres sous-groupes de médecins marginalisés sont plus avancés dans ce travail. Ainsi, certains médecins généralistes (Aliaud, 1987) et les homéopathes et acupuncteurs (Chateauraynaud, 1986, Traverso, 1993) ont entamé un travail de redéfinition de certains aspects de ce que doit être une bonne pratique médicale (refus de l'hyper-spécialisation, approche globale...). La mise en place de syndicats, de formations et de diplômes spécifiques ont joué un rôle important dans ces mouvements qui n'ont pas leur équivalent pour la médecine militaire.

Dans "l'enquête 1992", les principales revendications des médecins d'unité portent sur la reconnaissance de leurs compétences et de leur identité spécifiques par la direction du SSA à laquelle est reprochée la pratique d'un double langage : alors que le travail de médecine 
globale du médecin d'unité est loué dans les discours, dans la pratique, l'avancement et les postes les plus élevés sont réservés aux spécialistes et notamment aux agrégés. La médecine d'unité n'est pas alors une nouvelle spécialité, mais au contraire l'absence de spécialisation, signe d'infériorité, et les médecins d'unités sont perçus comme ceux qui n'ont pas réussi l'internat ${ }^{20}$. C'est ce que résume un des médecins interrogés : "le médecin militaire d'unité est assimilé à un officier de santé spécialisé, voire à un super infirmier; son choix n'est pas considéré comme une manière différente d'exercer son métier de médecin mais comme la traduction de son incapacité de passer un concours hospitalier" (LR.92). En l'absence de mouvement social organisé en faveur de la promotion de la médecine d'unité, les récriminations à l'encontre de la hiérarchie du SSA et des spécialistes des hôpitaux des armées, bien plus fortes que celles à l'encontre des spécialistes civils, offrent une certaine forme de protection de l'identité personnelle face à une position dévalorisée à la fois au sein du SSA et dans l'ensemble de la profession médicale. Certaines valeurs de la médecine civile, notamment la critique du salariat et de l'organisation bureaucratique, peuvent être mobilisées pour une remise en cause symbolique de la hiérarchie de la part de médecins d'unités cherchant à se construire une identité positive. Les rapports de force internes à la médecine militaire tendent alors à brouiller, dans certains cas, le lien entre les pratiques réelles et les représentations médicales affichées: quelques spécialistes plaident pour une médecine globale tandis que certains médecins d'unité peuvent se faire les défenseurs des principes dominants dans la médecine civile. Cette ambiguïté identitaire tend à s'estomper après l'étape de la reconversion qui opère ainsi comme un catalyseur des identités potentielles.

\section{II. - La reconversion dans le civil et la cristallisation des identités professionnelles}

Les limites d'âge et les choix de carrière (retour à la vie civile quand est acquis le droit à pension immédiat, recherche d'une rémunération plus élevée...) font que la majorité des médecins militaires terminent leur vie professionnelle dans le civil. Ils peuvent alors reprendre les normes et valeurs dominantes dans la médecine civile ou, au contraire, affirmer leur attachement aux particularités qui sont plutôt celles de la médecine d'unité et, ainsi, se construire une identité professionnelle spécifique ayant pour principe le retournement des stigmates (médecine salariée et bureaucratisée, traitement des problèmes sociaux, etc.). Les contraintes et les opportunités rencontrées lors de la reconversion vont jouer un grand rôle dans le choix de l'une ou l'autre de ces stratégies identitaires.

\section{1-Stratégies et pratiques de reconversion des médecins militaires}

\footnotetext{
${ }^{20}$ Cette idée n'est d'ailleurs pas totalement fausse puisque seuls $27,4 \%$ des médecins d'unité disent ne pas être intéressés par la préparation d'un concours d'assistanat.
} 
Les différentes études menées sur le retour dans le civil des anciens militaires (Duchamp, 1990 ; Loriol et Rio, 1994) montrent que deux facteurs favorisent particulièrement une bonne reconversion (délais de recherche réduit et rémunération avantageuse) : une spécialisation technique précise (par exemple pour les mécaniciens de l'armée de l'air) ou une valorisation de l'expérience militaire (par exemple, pour prendre deux cas très différents, le gardiennage pour les anciens gendarmes, ou les généraux intégrés dans les conseils d'administration des entreprises d'armement pour leur "carnet d'adresses"). Ces deux éléments se retrouvent dans les stratégies de reconversion des anciens médecins des armées. Les choix effectués sont donc conditionnés, pour une part, par l'expérience militaire. Une rapide typologie des types de carrière et de reconversion peut alors être esquissée à partir des résultats de "l'enquête 1994" auprès de médecins ayant quitté l'Armée depuis moins de trois ans.

Un premier type de carrière est représenté par les médecins militaires qui ont effectué toute leur activité professionnelle au sein du SSA. Il s'agit plutôt de spécialistes qui ont occupé des postes de responsabilité au sein de la Direction Centrale du SSA ou de médecins généralistes dont les opportunités de reconversion dans le civil auraient été peu avantageuses. Viennent ensuite les médecins qui se sont reconvertis grâce à la possession d'une spécialisation valorisée et recherchée dans le civil. D'après le médecin général Miné, directeur central en 1990 du SSA, ces spécialités sont le plus souvent: l'anesthésie-réanimation, la radiologie, l'ophtalmologie et l'ORL ${ }^{21}$. Leur reconversion se fait surtout dans le secteur libéral ou en clinique privée et les mobiles financiers semblent importants dans le choix d'une reconversion plus précoce. Ces médecins estiment le plus souvent que leur statut d'ancien militaire ne les a pas aidé pour leur reconversion mais n'a pas non plus constitué un handicap. Enfin, le troisième grand type de stratégie de reconversion est constitué par les médecins qui ont cherché à valoriser leur expérience spécifique de la médecine militaire pour se faire une place au sein la médecine civile. Il s'agit le plus souvent de généralistes ayant essentiellement une expérience de médecine d'unité. Les difficultés pour se constituer une clientèle en médecine générale ou pour racheter un cabinet à partir d'un certain âge (où il devient difficile d'obtenir un prêt bancaire) orientent plutôt ces médecins vers une pratique salariée. Les compétences en médecine du travail, en épidémiologie et dans la gestion d'institutions médicalisées (maisons de retraite, centres de convalescence...) sont les plus souvent mises en avant dans la recherche d'emploi. La spécialisation dans certains domaines de recherche comme l'étude de la fatigue et du stress (Loriol, 1995) ou l'alcoologie permet à quelques anciens médecins militaires d'occuper une position dominante dans ces secteurs un peu marginalisés de la recherche médicale. Ces médecins agissent alors comme de véritables

\footnotetext{
21 Cité dans Le quotidien du médecin du 30/01/90.
} 
"entrepreneurs de morale"22 militant en faveur d'une intégration des problèmes sociaux au domaine d'intervention de la médecine (Loriol, 1993).

Les compétences spécifiques que peuvent chercher à valoriser les anciens médecins militaires ne se limitent pas à la simple connaissance de certains sujets particulièrement étudiés dans les armées mais recouvre également tout le domaine des compétences organisationnelles. Ainsi, les possibilités de reconversion comme médecin-chef d'une maison de retraite sont non négligeables. Du fait de l'expérience en administration/gestion et en médecine communautaire, l'ancien médecin des armées a certaines facilités pour intégrer ce secteur. Cela lui permet, par surcroît, une spécialisation en gérontologie, domaine de recherche en construction où, comme dans le cas de la fatigue, la concurrence des grands médecins spécialistes reste faible. Ce type de spécialisation offre donc de bonnes opportunités pour des médecins occupant une position dévalorisée au sein de la profession.

D'après "1'enquête 1994", 69 des 112 médecins interrogés (soit 61,5\%) ont exercé une activité rémunérée après leur départ de l'armée. Grâce au croisement de plusieurs informations sur le premier emploi après le départ des armées (déclaration de la profession, code de la CSP, secteur d'activité, forme juridique, taille de l'entreprise) il est possible de distinguer des types particuliers de reconversion. Tout d'abord, sur les 69 médecins ayant réalisé une reconversion professionnelle, 37 ont choisi la pratique libérale. Ce chiffre est important, mais il faut rappeler ici que l'exercice libéral est le mode d'exercice majoritaire en France. Sept médecins seulement se sont orientés vers l'hôpital public, ce qui peut s'expliquer pour au moins deux raisons: d'une part le fait de ne pouvoir obtenir les postes les plus prestigieux, le début de carrière ayant été réalisé en dehors de l'hôpital public, et d'autre part le désir de conserver la pension militaire qui ne peut être cumulée avec un traitement public. Les 25 restants se sont orientés vers une pratique salariée de type médico-social. Parmi ceux-ci, près des deux-tiers sont des médecins du travail auxquels s'ajoutent deux spécialistes des ressources humaines en entreprise, deux fonctionnaires de l'Organisation Mondiale de la Santé, des médecins de dispensaire, de médecine préventive et même un instituteur.

Quels sont les facteurs qui orientent les choix de reconversion? Tout d'abord l'activité principale exercée lors de la dernière affectation au sein du SSA qui était soit la médecine générale (30\% de la population totale), soit une spécialité médicale $(60 \%)$, soit des activités de gestion et administration (10\%). Les spécialistes se reconvertissent plus souvent dans le libéral et la médecine hospitalière, du fait de plus grands atouts pour ces secteurs. Les médecins gestionnaires sont les plus nombreux (en proportion) à ne pas se reconvertir, ce qui

\footnotetext{
${ }^{22}$ Eliot Freidson (1984) utilise la notion forgée par Howard Becker (1985) à propos des médecins qui cherchent à définir comme pathologique, donc devant faire l'objet d'une intervention, certains états, comportements ou situations qui n'étaient pas perçus comme tels auparavant.
} 
s'explique par le fait qu'il s'agit de médecins plus âgés et en fin de carrière. Enfin, ceux qui avaient une pratique générale se tournent plutôt vers la "médecine sociale". Ces déterminations en termes d'opportunités professionnelles apparaissent renforcées par les représentations de la pratique médicale. Ainsi, les médecins qui se sont reconvertis comme salariés avec une pratique de type médico-social sont aussi plus souvent ceux pour qui il existe une différence, toujours perçue comme positive, entre la médecine militaire et la médecine civile ${ }^{23}$. Il existe ainsi une bonne cohérence entre défense d'une médecine plus "sociale" et reconversion dans des secteurs liés à ce type de pratique. Cela semble suggérer que l'attitude favorable vis-à-vis d'une pratique proche de la "médecine sociale" (salariée et ouverte aux problèmes non-organiques) ne serait, pour une part, qu'une adaptation aux difficultés rencontrées pour se reconvertir dans un secteur dominant du monde médical. Cela évoque un processus mental que l'on a qualifier de "réduction de la dissonance cognitive" (Festinger, 1957) : personne ne peut supporter de façon durable une contradiction entre ses pratiques ou ses choix et son système de connaissances, de croyances et de représentations. Le plus souvent un individu dans cette situation va changer ses représentations pour réduire ou supprimer cette contradiction afin de préserver la cohérence de son identité personnelle et son estime de soi. C'est pourquoi la stratégie de retournement du stigmate de la médecine militaire est fortement dépendante du type de reconversion effectué.

2- Type de reconversion et retournement du stigmate: les conditions de la construction d'une identité spécifique durable

Si l'effet durable de la première expérience professionnelle sur le processus de construction identitaire commence à être connu (Baszanger, 1983; Dubar, 1991 ou Bouchayer, 1994), l'impact d'une reconversion, qui oblige le professionnel à s'adapter à de nouvelles formes de travail et de concurrence, reste peu étudié. Dans le questionnaire de "l'enquête 1994" destiné aux ex-militaires du service de santé, une question réservée aux seuls médecins demandait à ces derniers si leur expérience militaire avait eu une influence sur leur façon de concevoir la médecine de façon telle que leur conception soit différente de celle des médecins civils. En cas de réponse positive, les répondants étaient invités à exposer, en quelques lignes, dans quel sens avait joué cette influence (LR1.94, dont sont tirées les citations de ce paragraphe). Sur les 112 médecins interrogés, 52 se sont trouvés dans ce cas ${ }^{24}$. Dans tous les cas, sauf un, la ou les différences signalées étaient de nature positive et faisaient, d'un certain point de vue, de la pratique militaire une pratique supérieure à la

\footnotetext{
${ }^{23}$ Voire supra, paragraphe suivant, pour des détails sur cette question.

${ }^{24} 48$ pensent qu'il n'y a pas de différence entre leur conception de la médecine et celle des médecins civils et 12 ne se prononcent pas. Le refus de reconnaître une différence peut s'interpréter comme le refus de voir la médecine militaire, plutôt "dominée" sociologiquement par la médecine civile, prise pour une "sous-médecine", c'est à dire comme l'affirmation d'un même degré de qualité.
} 
pratique civile. Un seul médecin, en effet, se plaint, du caractère "lourd et bureaucratique" du service de Santé, aspect négatif de l'organisation et de la discipline vantées par d'autres ${ }^{25}$. Après le départ des armées, deux grandes stratégies identitaires sont possibles : refuser de reconnaître une différence entre la médecine militaire et la médecine civile et donc s'identifier aux valeurs dominantes de cette dernière, ou, au contraire, remettre en cause ces valeurs en présentant comme positifs les aspects stigmatisés par la médecine dominante.

Les médecins qui développent plutôt ce dernier type de stratégie se distinguent beaucoup plus par le style de reconversion réalisé que par la possession, ou non, d'une spécialité avant le départ des armées. C'est donc bien le moment de la reconversion qui permet de fixer, ou non, les identités construites au cours de la carrière, notamment lors du passage par la médecine d'unité. Il n'existe pas, en effet, de différences, dans les réponses, entre les généralistes et les spécialistes (qui pourtant n'ont connu la médecine d'unité qu'au début de leur carrière). Cela est d'autant plus étonnant que les spécialistes qui se sont reconvertis dans une pratique libérale sont moins enclins à développer une représentation de la médecine militaire comme différente et supérieure. Mais les spécialistes qui ne se reconvertissent pas dans le secteur libéral et ont fait une carrière longue dans l'Armée sont parmi les premiers à développer une identité professionnelle spécifique de médecin militaire, malgré leur spécialisation. Les généralistes qui se sont reconvertis dans des secteurs proches de la médecine sociale sont également les plus nombreux à défendre les valeurs stigmatisées par la médecine dominante.

Pour ceux qui revendiquent une différence entre la médecine militaire et la médecine civile, les réponses quant à la nature de cette différence présentent une bonne cohérence. Cela permet de penser que l'ensemble de ces petits discours offre un résumé fiable de l'identité professionnelle spécifique -c'est à dire la manière particulière d'interpréter leur rôle et leur activité- que certains médecins militaires ont réussi à actualiser dans leur reconversion. Cette identité se structure autour de trois grands thèmes qui sont autant d'occasions de remettre en cause certaines des valeurs dominantes dans la médecine civile : le "sens du service public" qui permet de critiquer le mercantilisme médical imputé implicitement à la médecine libérale du fait du paiement à l'acte, la "polyvalence des tâches", liée à la défense de l'étude par la médecine de certains problèmes sociaux et "l'expérience outre-mer" qui permet d'introduire l'idée d'une médecine moins coûteuse et plus humaine.

\footnotetext{
${ }^{25}$ Il faut aussi noter qu'un autre médecin militaire, ayant répondu négativement à la question -et n'ayant donc pas utilisé la première libre-réponse (LR1.94)- critique, dans la deuxième libre-réponse (LR2.94), le rôle humanitaire du service de Santé ("l'utilisation de l'Armée Française au service des barbus tiers-mondistes de la SDN (sic) devenait franchement insupportable"). D'autres médecins, plus nombreux, déplorent au contraire de ne pas toujours pouvoir se reconvertir dans la coopération ou les ONG (LR2.94)
} 
Les réponses les plus fréquentes (44) sont celles qui peuvent être regroupées sous la notion de "sens du service public", comme l'écrivent d'ailleurs littéralement deux des médecins interrogés. Celui-ci se traduit tout d'abord par l'affirmation de l'indépendance, notamment dans la relation malade-médecin, de la médecine militaire vis à vis de l'argent : "moins de liens avec l'argent", "médecine désintéressée", "exercice de la médecine libéré du problème financier", etc. Le "sens du service public" se traduit également dans plusieurs réponses par la revendication d'une plus grande "disponibilité" vis-à-vis du malade, car le dévouement - et non le gain monétaire - serait la première motivation du médecin militaire. Le désintéressement va de pair, pour les médecins interrogés, avec un sens plus poussé du bien public pour au moins deux raisons : tout d'abord, le médecin militaire n'étant pas motivé par une augmentation de ses revenus peut se consacrer plus complètement aux exigences de la médecine et de la santé publique ("le médecin militaire étant dégagé de toute contingence matérielle peut s'adonner totalement à la pratique de son art"). Ensuite, se sentant moins soumis à la pression de la clientèle, il estime être plus libre pour agir au mieux selon le bien public et celui du patient ("plus grande aisance intellectuelle, puisque non-assujetti aux impératifs d'une clientèle"). Cette "liberté" est pour plusieurs médecins le gage d'une plus grande rigueur dans le diagnostic et le traitement ("plus de rigueur dans l'approche diagnostique"; "grande liberté dans l'exercice et rigueur dans l'organisation"). Cette rigueur est aussi le fait, dans l'esprit des personnes interrogées, de l'organisation bureaucratique, centralisée et hiérarchisée qui permet d'éviter notamment les gaspillages ("moindre prescription d'examens complémentaires"). Le "sens de l'économie" (comme il est possible de le lire dans un des questionnaires) est en effet un autre des attributs du "sens du service public" revendiqué par les médecins militaires ("la modération dans les prescriptions"; "économies de santé"...).

Le "sens de l'économie" peut être rattaché au deuxième grand groupe de réponses (23) centré sur la notion de "polyvalence des tâches". C'est parce qu'ils ont à la fois un rôle de gestionnaire et de soignant que certains médecins militaires affirment leurs préoccupations en économie de la santé ("gestion des dépenses de santé, ayant été la plupart du temps prescripteur de soins et gestionnaire de budget"). Le médecin militaire se caractériserait en effet par une "formation polyvalente avec des expériences différentes touchant plusieurs facettes de la médecine : aptitudes, médecine générale, expertise, médecine du travail, contrôle médical...". Cette richesse des activités est liée aux rôles nombreux du médecin militaire, mais aussi à la fréquence des mutations et des changements des fonctions exercées. De cette "richesse des expériences", résulte une vision particulière de la médecine qui comprend notamment : une "médecine sociale" et "collective" ("envisager la médecine dans son ensemble, médecine individuelle + médecine collective"), une approche écologique ("connaissance d'un milieu et des contraintes d'un environnement particulier: 
l'aéronautique", "prise en compte du malade dans la totalité de son milieu"), une "médecine préventive" etc. Le lien avec le "sens du service public" se fait également à travers la "dimension santé publique", revendiquée dans plusieurs réponses, la prise en compte du bien collectif et la recherche d'économies en matière de santé. La polyvalence des tâches permet de dépasser les seuls intérêts individuels.

Enfin, le troisième thème sous lequel peuvent être regroupées les réponses (11) est celui de "l'expérience outre-mer" que la plus grande partie des médecins militaires ont connu au cours de leur carrière ("ouverture sur le tiers-monde", "lutte contre la maladie à l'échelon international"...). Ce thème peut d'ailleurs être rattaché au précédent puisqu'il est également révélateur de la diversité d'expérience du médecin militaire. De cette expérience, les médecins interrogés déclarent tirer des leçons pour leur pratique et leur conception de la médecine en France métropolitaine : "expérience outre-mer : meilleur regard sur les problèmes métropolitains"; "l'exercice de la médecine dans le tiers-monde (Afrique), conduit à relativiser certaines priorités". Ce genre de discours n'est pas loin de rappeler celui tenu, dans le cadre de la critique de l'institution médicale, sur les "médecins aux pieds nus" chinois, symbole d'une médecine moins coûteuse pour une utilité sociale équivalente (par exemple, Illich, 1975). La préoccupation au sujet des économies de santé peut donc être décelée dans cette troisième thématique. De même, à travers l'aspect "d'action humanitaire" que revêt l'intervention dans les pays du tiers-monde apparaît l'idée de goût du "bien public" et d'humanisme ("nous avons toujours été les humanitaires, sans avoir jamais oublié d'être humain").

Si l'humanisme, le désintéressement, le dévouement sont des valeurs également revendiquées par la plupart des médecins libéraux et hospitaliers civils, le discours des anciens médecins militaires cités se singularise néanmoins par deux points notables. Tout d'abord par l'idée qu'il peut être acceptable de privilégier, dans certains cas, la satisfaction de besoins collectifs (économies de santé, besoins de l'institution militaire, santé publique, etc.) par rapport aux intérêts individuels des malades; ce point est particulièrement délicat puisqu'il constitue un des axes possible de remise en cause de la médecine militaire par les médecins civils. Enfin, par l'affirmation que le fonctionnariat peut être un facteur d'efficacité et d'indépendance dans la pratique médicale. Les médecins interrogés semblent ici reprendre à leur compte l'idéal-type weberien de la domination bureaucratique efficace et rationnelle, reposant sur des règles strictes : hiérarchie des compétences, contrôle par les textes, discipline, etc. (Weber, 1969). Aux critères de Max Weber, les médecins interrogés ajoutent l'idée de dévouement et de service au public. Ce type d'identité professionnelle peut-être rapproché de "l'identité de service public" dont le renouveau est diagnostiqué par certains 
(Francfort et autres, 1995). Ce renouveau pourrait être analysé comme une réaction - en forme de renversement du stigmate - à la dévalorisation, dans l'opinion, de l'image du fonctionnaire.

Cette vision enchantée des caractéristiques habituellement stigmatisées de la pratique médicale militaire, défendue par la moitié des anciens médecins militaires interrogés, contraste singulièrement avec les critiques adressées par certains des médecins d'unité de "l'enquête 1992". Pour expliquer cette apparente contradiction, il est possible de faire l'hypothèse, malheureusement non vérifiable, que les médecins qui se montrent les plus critiques à l'égard des caractéristiques propres à la médecine militaire (salariat, prise en charge de certains problèmes sociaux, etc.) sont aussi ceux pour qui il n'existe pas de différences entre la médecine militaire et la médecine civile. Ainsi, les anciens médecins des armées qui perçoivent positivement les spécificités de la pratique militaire en font le support d'une véritable identité professionnelle différente de celle du médecin civil, alors que ceux qui ont une vision plus négative de ces mêmes spécificités n'en font pas l'essence propre de la pratique militaire mais de simples conditions défavorables qui perturbent, sans la changer fondamentalement, la nature de leur travail. Après le retour dans le civil, les médecins qui n'ont pas eu à s'insérer dans la médecine civile parce qu'ils avaient quitté tardivement le SSA et prenaient directement leur retraite ou ceux qui ont su utiliser leur expérience militaire pour leur reconversion ont pu également oublier les griefs qui les opposaient aux représentants de l'organisation du SSA pour construire leur identité non plus contre ces représentants mais contre la pratique dominante dans la médecine civile.

L'identité professionnelle du médecin militaire se construit donc, tout au long de sa carrière avec quelques moments clés (études, expérience en unité et outre-mer, reconversion dans le civil) sans que des déterminismes précoces ne puissent être mis en évidence. Ainsi, le fait d'obtenir une spécialité ne prédispose pas forcément à l'acceptation du modèle médical dominant. Tout dépend en fait de la façon dont sera utilisée par la suite cette spécialité dans les choix de carrière : si la spécialisation est un moyen de faire carrière au sein du SSA, elle sera plutôt un facteur d'attachement, au moins dans les discours, aux spécificités de la médecine militaire ; au contraire, si la spécialisation est utilisée pour opérer une reconversion avantageuse dans le civil, elle sera fortement liée à un rejet de la pratique salariée et de la médicalisation de problèmes non strictement organiques. A chacun des moments clés de sa carrière, le médecin militaire se trouve confronté à un système de contraintes et d'opportunité (impossibilité du paiement à l'acte, mais possibilité de mener des recherches nouvelles, possibilité d'emploi dans une entreprise comme conseiller en ressources humaines ou possibilité d'une pratique libérale rémunératrice, etc.) qui vont le pousser à prendre position 
par rapport aux valeurs dominantes dans la médecine civile et à faire des choix déterminants. Si ces choix sont sans doutes liés aux expériences passées et aux choix précédants, ils participent au sens que prendront ces derniers par la suite. Le rapport au système normatif dominant reste central mais ils ne peut pas être ramené à une simple équation reliant les positions dominées à des prise de position dissidentes et les positions dominantes à des prises de position conformistes. Le médecin militaire, comme d'ailleurs le médecin du travail, malgré sa position dominée se veut médecin avant tout. Cela le conduit parfois à rejeter le système de normes dominant au sein de la médecine civile et parfois à y adhérer

L'hypothèse que le médecin militaire construit son identité avant tout en tant que médecin plutôt qu'en tant que militaire est peut-être liée au parti-pris de cet article de se fonder sur une problématique née des travaux de sociologie de la profession médicale, mais elle rejoint également les discours des médecins lus ou rencontrés. D'ailleurs si la crainte d'être accusé d'être un mauvais médecin est sous-jacente dans beaucoup des prises de position analysées, celle d'être accusé d'être un mauvais militaire n'apparaît pas. Certes, l'appartenance à l'Armée offre un contexte social fort pour l'orientation des conduites du fait de la valorisation de certaines valeurs dans cette institution (hiérarchie, discipline, tradition...). Les remarques faites dans les paragraphes précédents sur l'organisation, la rigueur ou le bien public comme valeurs de la médecine militaire peuvent renforcer cette impression. Mais ce qui caractérise le SSA, c'est aussi son autonomie professionnelle par rapport au reste de l'Armée. La plupart des travaux de sociologie militaire, tant français (Thomas, 1981) qu'américains (Janowitz, 1960), décrivent les métiers des armes comme tiraillés entre deux types-idéaux : celui de la vocation militaire, totalement coupée de la vie civile du fait de la spécificité guerrière et celui du technicien en uniforme dont les normes professionnelles sont les mêmes que celles de ses homologues civils. Les médecins militaires sont, parmi les militaires de carrière, les plus proches du second modèle. Comme le note le médecin général Debénédétti (1967, p. 103), "si le médecin militaire est soumis à la discipline militaire dans l'exercice de la part purement militaire de ses fonctions, il jouit du point de vue technique, d'une indépendance absolue. Qu'il s'agisse de médecine curative, de médecine préventive ou de médecine d'expertise, le médecin militaire ne relève que de sa conscience. Il convient de mettre avec vigueur l'accent sur cette indépendance technique qui s'oppose à la discipline militaire pure. C'est là une condition essentielle de cette profession : elle lui confère une situation très particulière dans l'armée" ${ }^{\prime 2}$. Le caractère militaire du SSA peut favoriser une tendance à l'organisation et à la bureaucratisation, mais il ne s'agit pas du facteur principal pour rendre compte de la conception spécifique de la médecine développée par la majorité des médecins des armées.

26 C'est la caractéristique minimale d'une profession, pour Eliot Freidson (1984) que d'avoir une totale autonomie dans la partie technique de son travail. Dans ce cadre, les professionnels ne peuvent être contrôlés que par des professionnels. 
La médecine militaire ne peut pas être considérée comme un segment particulier de la profession médicale, au sens que donne Strauss (1992) à ce terme, car tous les médecins des armées ne développent pas une identité professionnelle favorable aux spécificités de la pratique militaire. De plus, ceux qui adoptent ce type d'identité se retrouvent sociologiquement proches, dans leur parcours et leurs représentations des omnipraticiens qui cherchent à fonder la spécificité de leur pratique (par exemple certains des médecins regroupés dans le syndicat MG France) et des certains des médecins salariés civils. Ces médecins militaires, d'ailleurs, effectuent toute leur carrière au sein du SSA ou se reconvertissent plus volontiers vers une pratique médico-sociale salariée. Leur carrière militaire, notamment l'expérience en médecine d'unité et parfois l'exercice outre-mer, leur ont permis de se forger une véritable identité professionnelle distincte et positive, prenant le contre-pied des valeurs dominantes dans la profession médicale (exercice libéral, spécialisation, approche centrée exclusivement sur le biologique et le physiologique...). Les autres médecins militaires, au contraire, refusent d'attribuer au médecin des armées une identité professionnelle propre, leur carrière au sein du SSA est plus courte et la cause de leur départ est souvent la recherche d'une rémunération plus importante dans le civi127. Ils alimentent ainsi le thème récurrent au sein du SSA du "malaise des médecins militaires", de la "désertion des spécialistes" 28 . Tout se passe comme si les médecins du premier groupe avaient accepté leur position particulière au sein de la médecine et avaient développé, principalement lors des premières expériences professionnelles, des représentations favorisant une adaptation à leur position dominée et la constitution d'une identité positive ${ }^{29}$. Les médecins du second groupe semblent au contraire réaliser une "socialisation anticipatrice" (Merton, 1965) par rapport aux représentations de la médecine civile dominante qu'ils espèrent intégrer. Ils n'ont donc pas besoin, en ce sens, de se construire une identité professionnelle positive de médecin militaire et gardent une vision plus critique vis-à-vis des spécificités du travail de médecin des armées. Les principes de différenciation des identités professionnelles à l'oeuvre parmi les médecins des armées sont donc identiques à ceux qui traversent la médecine civile et les quelques résultats proposés ici pourraient être utilement comparés avec ceux apportés par d'autres sous-groupes dominés de médecins (médecins du travail, médecins pénitentiaires, etc.).

\footnotetext{
27 D'après "l'enquête 1994", 74\% des anciens médecins militaires qui se sont reconvertis dans le civil déclarent que le total annuel de leurs revenus actuels (y compris éventuellement la pension militaire) est supérieur à leur dernière solde annuelle ; pour $42 \%$ ce total est même plus d'une fois et demi supérieur à la dernière solde.

28 Voir, par exemple, Le Quotidien du médecin du 30/01/1990.

${ }^{29}$ Le caractère "adaptatif" des représentations sociales a souvent été souligné par les psychologues sociaux : "Les représentations sociales sont des modalités de la pensée pratique orientées vers la communication, la compréhension et la maîtrise de l'environnement social, matériel et idéel" (Jodelet, 1984), "Les représentations sociales sont des principes générateurs de prises de position liées à des insertions spécifiques dans un ensemble de rapports sociaux et organisant les processus symboliques intervenant dans ces rapports" (Doise, 1986).
} 


\section{BIBLIOGRAPHIE}

Arliaud M., 1981, L'enjeu d'une lutte professionnelle : Discours technique et pratique sociale, colloque de sociologie du travail et de la santé, Paris, C.N.R.S., 165-182.

Arliaud M., 1987, Les médecins, Paris, La découverte, Coll. Repères, 128 p.

Baszanger I., 1983, La construction d'un monde professionnel : entrée des jeunes praticiens dans la médecine générale, Sociologie du travail, 3, 275-294.

Becker H., 1985 (première ed. 1963), Outsider. Etudes de sociologie de la déviance, Paris, A.M. Métailié.

Bouchayer F., 1984, Médecins et puéricultrices de Protection Maternelle et Infantile, Revue Française de Sociologie, XXV, 1, 67-90.

Bouchayer F., 1994, Les voies du réenchantement professionnel : le choix des médecins généralistes praticiens des médecines hétérodoxes, dans Aïach P. et Fassin D. (dir.), Les métiers de la santé, Paris, Anthropos.

Brumter C., 1979, La participation du Service de santé des armées à la protection sanitaire de la Nation, Revue française des Affaires sociales, 3, 187-204.

Chateauraynaud F., 1986, Les médecins et les techniques de soin non-allopathiques, Sciences Sociales et Santé, IV, 3, 5-49.

Conseil de la fonction militaire du Service de santé des armées, 1993, Résultats de l'enquête effectuée auprès des médecins d'unité en octobre 1992, Paris, Direction centrale du Service de santé des armées, Ministère de la Défense, $86 \mathrm{p}$.

Cristau P., Tardy D., 1989, L'opinion des élèves de l'école du SSA sur l'alcoologie, Médecine et Armées, XVII, 1, 51-56.

Debénédéti R., 1967, La médecine militaire, Paris, PUF, Coll. "Que sais-je ?", 128 p.

Doise W., 1986, Les représentations sociales : définition d'un concept, dans Doise W. et Palmonari A., Les représentations sociales, un nouveau champ d'étude, Genève, Delachaux et Niestlé

Dubar C., 1991, La socialisation, Paris, Armand Colin.

Duchamp J.L., 1990, Mobilité, stratégie et reconversion : le retour à la vie civile des cadres militaires titulaires d'une pension de retraite, Paris, Thèse de sociologie sous la direction de J. Lautman, Université de Paris V, $469 \mathrm{p}$.

Dyevre P. et Giacomoni A., 1995, Médecine du travail dans les armées : organisation, données statistiques, et aspects méthodologiques, Médecine et Armées, XXIII, n. 2, 115-120.

Ferrand-Nagel S., 1993, Médecine libérale, médecine sociale et régulation, Revue française des Affaires sociales, 2, 43-79.

Festinger L., 1957, A theory of cognitive dissonnance, New-York, Harper \& Row.

Forissier R., 1988, Les 100 ans d'existence des écoles du SSA, Médecine et Armées, XVI, 5, 383-396.

Francfort I., Osty F., Sainsaulieu R. et Uhlade M., 1995, Les mondes sociaux de l'entreprise, Paris, Desclée de Brouwer.

Foucault M., 1963, Naissance de la clinique, Paris, PUF, 215 p.

Freidson E., 1984 (première éd. 1970), La profession médicale, Paris, Payot, 369 p.

Gateff G., Le Bras J., Bourrel P., 1986, Santé communautaire et milieu militaire : réalité ou utopie ?, Médecine et Armées, XIV, 1, 17-23.

Gofmann E., 1968 (première ed. 1961), Asiles. Etude sur la condition sociale des malades mentaux, Paris, Les Editions de Minuit, $447 \mathrm{p}$.

Hassenteufel P., 1997, Les médecins face à l'Etat. Une comparaison européenne, Paris, Presse de Science Po., $367 \mathrm{p}$.

Herzlich C., Bungener M., Paicheler G., Roussin P., Zuber M.C., 1993, Cinquante ans d'exercice de la médecine en France : carrières et pratiques des médecins français, 1930 - 1980, Paris, INSERM Doin, $274 \mathrm{p}$.

Hugues E.C., 1958, Men and their work, Glencoe, The Free Press.

Illich I., 1975, Némésis médicale, Paris, Seuil.

Janowitz M., 1960, The professionnal soldier : a social and political portrait, New-York, The Free Press. 
Jodelet D., 1984, Reflexions sur le traitement de la notion de représentation sociale en psychologie sociale, dans "Les représentations sociales", dirigé par Schiele B. et Belisle C., Communication, Information, VI, 15-41.

Loriol, M., 1993, La médicalisation des problèmes sociaux, l'exemple de la fatigue, mémoire de DEA sous la direction d'A.M. Guillemard et C. Horellou-Lafarge, Université de Paris I, 123 p.

Loriol M., Rio B., 1994, Les conditions de retour à la vie civile des militaires ayant acquis droit à pension, Paris, Obsevatoire Social de la Défense, Ministère de la Défense, $111 \mathrm{p}$.

Loriol M., 1995, Construction de la maladie et influence professionnelle : l'exemple de trois approches de la fatigue, Sciences Sociales et Santé, XIII, 3, 103-130.

Merton R., 1965 (première éd. 1949), Eléments de théorie et de méthode sociologiques, Paris, Plon, Coll. Recherches en sciences humaines, $514 \mathrm{p}$.

Piotet F., Correia M., Lattès C. et Moisan A., 1997, Enquête sociologique. Entre profession et métier : les médecins du travail, Archives des maladies professionnelles et de médecine du travail, CVIII, 1, 22-89.

Sainsaulieu, R., 1977, L'identité au travail, Paris, Presses de la FNSP, 487 p.

Strauss A.L. (textes réunis et présentés par Isabelle Baszanger), 1993, La trame de la négociation, Paris, L'Harmattan, Coll. Logiques Sociales, 311 p.

Thomas H. J-P., 1981, Fonction militaire et système d'hommes, Paris, Fondation pour les études de la défense nationale.

Tournier-Lasserve C., 1982, Reflexions sur le système de santé appliqué aux armées, Médecine et Armées, X, 4, 375-382.

Traverso D., 1993, La pratique médicale alternative: L'expérience de l'homéopathie et de l'acupuncture, Sociologie du travail, 2, 168-198.

Weber M., 1969, Economie et société, Paris, Plon, 660 p. 